\title{
A comparison of existing transformation models to improve coordinate conversion between geodetic reference frames in Nigeria
}

\author{
Kalu Ikechukwu ${ }^{\mathrm{a}}$, Christopher E. Ndehedehe ${ }^{\mathrm{b}}$, Onuwa Okwuashi ${ }^{\mathrm{c}}$, Aniekan E. Eyoh ${ }^{\mathrm{d}}$ \\ ${ }^{a, c, d}$ Department of Geoinformatics \& Surveying, University of Uyo, P.M.B. 1017, Uyo, Nigeria \\ ${ }^{\mathrm{b}}$ Australian Rivers Institute and Griffith School of Environment \& Science, Griffith University, Nathan, \\ Queensland 4111, Australia
}

\begin{abstract}
Efficient transformation parameters are key to effective geodetic operations between any systems. With the advancement in technology, an improved interaction between geodetic reference frames has been noticed irrespective of their inherent heterogeneous deformations and the intermittently present oversight that exist as a result of the conversion methodology. The spatial data captured using the Global Navigation Satellite System (GNSS) has a reference datum based on the World Geodetic System 1984 (WGS84) ellipsoid. These data usually require a transformation to a local projection with its ellipsoid and datum and vice versa. These geodetic operations are unavoidably vulnerable to data loss due to distortion, especially if the applied model is unsuitable for the transformation between reference frames. Therefore, the main aim of this study is to authenticate the existing set of parameters for the local and geocentric systems in Nigeria by assessing the efficacy of the Bursa Wolf (BW) and the Molodensky Badekas (MB) models. To this end, both models (BW and MB) are compared in this study and used in the development of the Helmerts seven transformation parameters between Minna datum (Clarke 1880) and WGS84 reference ellipsoids for a large region in Southern Nigeria. Results show that the sets of datum shift transformation parameters for both models (a scale factor with three sets of translational and rotational parameters) derived from the exercise of the BW and the MB models revealed a high degree of correlation between the expected and derived set of coordinates during the validation/testing phase using each set of models. The validation exercise was carried out on a total of seven points, which were not part of the original computations. These points were distributed across the region to provide a better framework anda higher confidence level. Overall, an improvement of $68 \%$ is observed in terms of the correlation between expected and derived coordinates in the validation pointsand from the obtained root mean square values. Ultimately, the MB model is preferred to the BW model evidently because the transformation involves a global-local reference frame.
\end{abstract}

Keywords: $\quad$ Datum, GNSS, Bursa Wolf, UTM, GPS 


\section{Introduction}

Helmerts definition of geodesy $(\gamma \eta=$ Earth, $\delta \alpha \omega \omega=1$ divide $)$ as the science of the measurement and mapping of the earth's surface aims at the establishment of a framework of finely distributed points which are otherwise referred to as geodetic control points. The coordinates of the points formed on this framework are computed extensively with a geodetic datum which serves as its reference. Transformation parameters exist to help with relating different geodetic reference systems but are usually restricted because of the heterogeneous and local character of deformations caused by the procedures adopted in classical networks (Krakiwsky et al. 1974). As an illustration, Figure1a explicitly shows the uniqueness of a point on the earth's surface based on the reference frame in use. When using the approach of conformal transformation for coordinates between datums (local and global), errors that may exist in the local datums are simply transformed to the global datums, thereby causing reduction in accuracy to the entire system state (Featherstone 1997).

Several countries around the globe have adopted different ellipsoids in a bid to simplify the science of surveying and mapping in their respective regions (Fig. 1b). In regions where topocentric datums have been introduced, datum transformation becomes inevitable. This was the basis for the transformationbetween Australian datums and the empirical comparison of coordinate transformations between the Australian Geodetic Datum and Geocentric Datum of Australia (Kinneen \& Featherstone 2004; Featherstone et al.1997, 1999). However, as with other regions with a relatively less accurate geocentric datum fit, this is the case with Nigeria whose local coordinate system is the Minna datum, which is based on the Clarke 1880 ellipsoid. The conversion between coordinate values associated with, for example a datum 'A' to another datum 'B' usually use 3D transformations (commonly referred to as datum transformation) (Deakin 2006). This conversion is achieved from the rotations that occur between the two 3D coordinate systems which can be in minutes (usually less than 1 second of arc). However, certain approximations are used to simplify rotation matrices; these simplified matrices are a common feature of the two models which will be explored in this study.

The assumptions for the Minna datum in Nigeria (Okeke 2013), have been used for mapping and coordinating of points across the country over a long period of time. This assumption poses a distinct challenge as it compromises the integrity of coordinate transformations from the local datum to the more precise global datum (WGS84). In turn, this diminishes the sole aim of creating a global datum, which is, the creation of an accurate geospatial data integration of coordinates around the globe. The oversight being experienced between the local Minna datum and global datum transformation parameters brings about an erroneous and inaccurate geospatial data system (Dzidefo 2011). This in turn tends to affect map users who rely on data from maps and other geoinformation systems which are based on the global coordinate systems as a result. For the most part, such effects are the results of faulty transformation technique. This has raised key concern among multi-national firms and mapping industries in Nigeria some of which have attempted to develop their own models in the process of executing their operations in the country.

It is obvious that over the years, the study of geodetic surveying in Nigeria has undergone significant improvement and changes; the mode of data acquisition has long metamorphosed as well as

the coordinate system from the regional Clarke 1880 to the WGS84 (global) system due to the advent of the Global Navigation Satellite System (GNSS). This advancement has necessitated a more thorough 
verification of the parameters being used for coordinate conversions of certain regions by validation using different models, algorithms, and computations.

Preceding the advent of the GNSS system, many regions including Nigeria adopted a local reference frame to provide the network of permanent ground reference points and the associated intellectual and positional data to enable and ensure that data concerning land, resources, and location is managed in an orderly and systematic manner (Ayer 2008). But times have changed as the Global systems are now predominant and the advent of the Global Positioning System (GPS) and the likes, which aims at providing a unified system of the entire earth (relatively poor in accuracy as it may seem) are now emerging (Dawod et al. 2011; Ziggah et al. 2013, 2019). This, therefore, calls for the need for each region, especially developing economies with limited resources to adopt means of transforming their local coordinate systems to the global systems for integration into the global systems. If it were simple, widely used existing geodetic controls, would be re-observed, leveraging on the global system. But this could be considerably inefficient and poor use of human resources. The key question therefore is how and what is the best way to relate both systems? This question is one of the important motivations warranting this study.

In this study, the main aim is to establish a geodetic relationship between a global and local ellipsoid using the Euclidian similarity transformation which can relate two non-deformable Cartesian reference frames via seven parameters. To this end, two novel and well-known models were used in the creation of these seven parameters, which will serve as a strong tool for unification among the coordinates systems in Nigeria. The accuracies of these models (Molodensky Badekas and Bursa Wolf) were tested against each other by a way of comparison between them. The network used for the creation of this model was slightly dense and spread uniformly across the region in question. These controls were established in the year 2002 by the regional government in Southern Nigeria for orthomapping. This project required the establishment of first order controls using the Trimble differential GPS and the data was obtained using the static mode. Details about the data and methodology employed are further explained in the remaining sections of this manuscript.

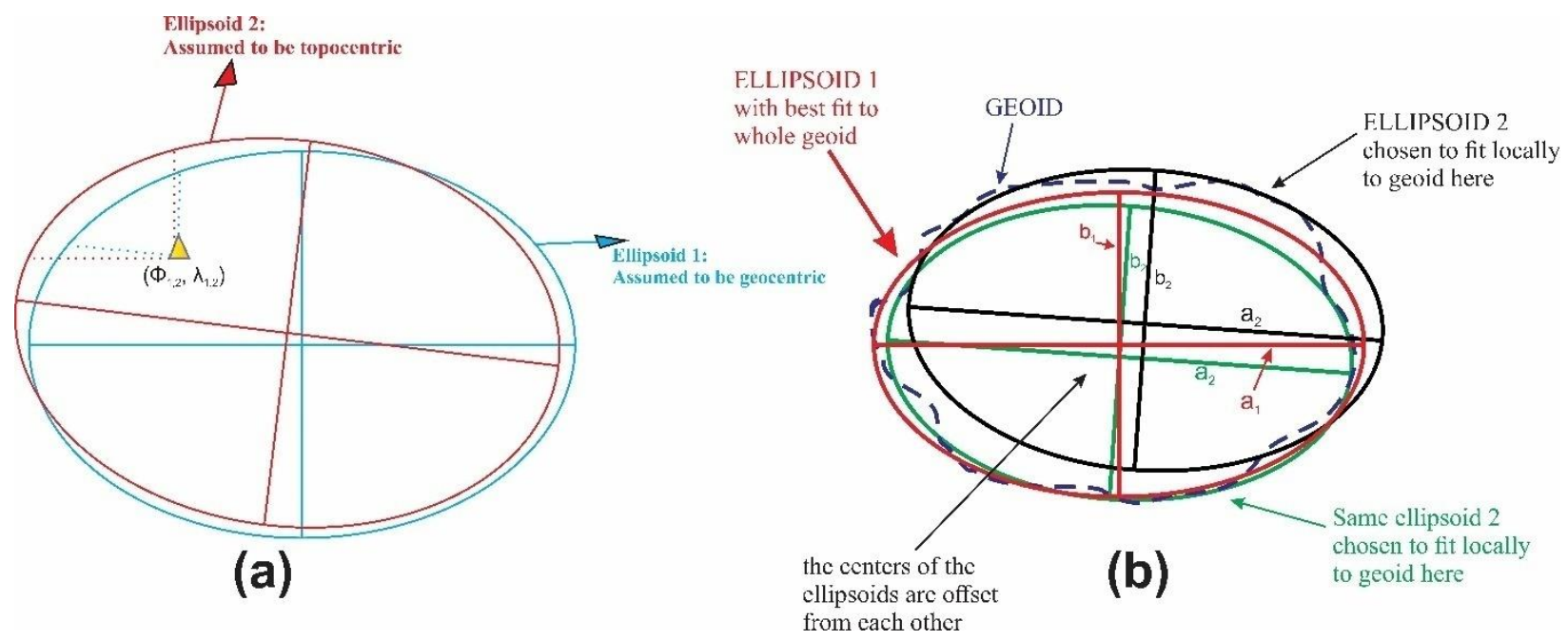

Figure 1: The uniqueness of a point on the earth's surface based on a reference ellipsoid and several ellipsoids adopted by several regions to fit locally to their geoid.

\section{Datums and geodetic reference systems}




\subsection{Understanding datums and projections}

Geodetic datums are referred to as curved surfaces where the positions of points are referenced to; while the vertical positions of points (orthometric height, $\mathrm{H}$ ) are referenced to the means sea level (Heiskanen1967). However, heights in terms of a geodetic datum will be in relation to an ellipsoid. Geodetic datums are broadly classified into two categories, the geocentric and the local. The main aim of the geocentric datum (Fig. 2a) is to present on average, a good fit of the entire earth and as such does not seek to be a perfect approximation for any single part of the earth. Examples of this include; the WGS84 ellipsoid, GRS80 ellipsoid, International Terrestrial Reference Frames (ITRF), etc. Local geodetic datum, on the other hand, is designed specifically to approximate the size and shape of a particular part of the earth's sea-level surface (Fig. 2b). Several national local ellipsoids were based on local geodetic datums until the introduction of satellite systems. Another type of coordinate system is provided by map projection (Janssen 2009).

(a)

Fits this part of

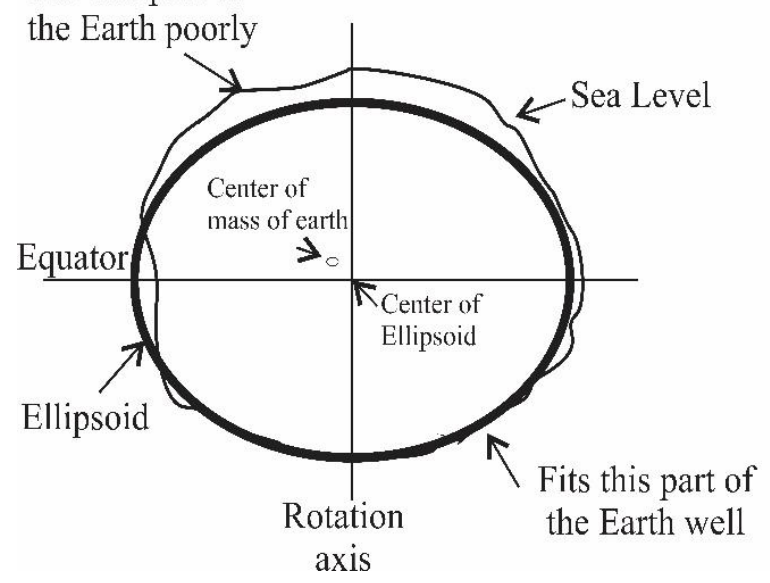

(b)

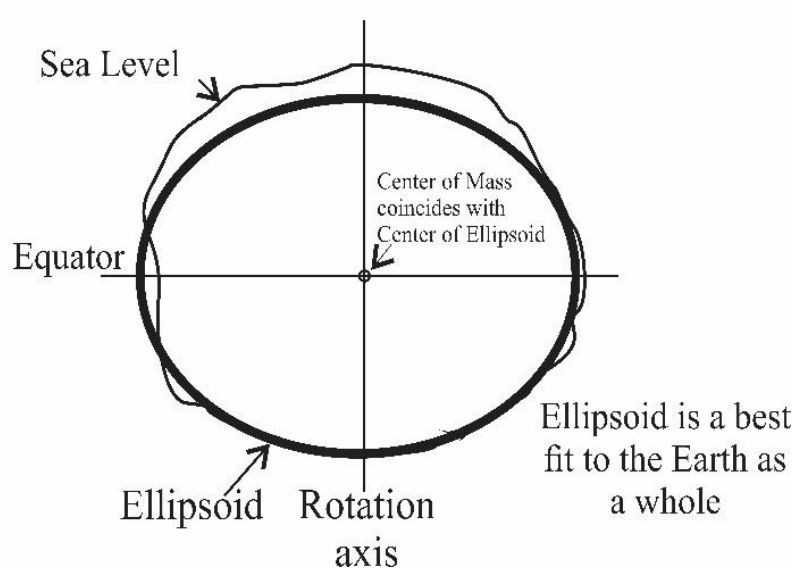

Figure2: Geocentric datum showing generic fit to the earth as a whole and Local geodetic datum showing the best fit of a particular part of the earth.

\subsection{Geodetic reference frames and systems}

The Geodetic reference system is only a mathematical concept whose feasible realization (materialization) through geodetic observations is the geodetic reference frame. The orientation and origin of the reference system for a particular epoch are being defined by the geodetic frame positioned with the Earth (i.e. as the ITRF). In geodesy, a geometric feasible reference model is a revolution ellipsoid, which is defined by two parameters; usually, the semi-major axis, $a$ and the flattening $f$ (Vanicek et al.1986). The coordinates of a point $\mathrm{P}$ related to the reference ellipsoid are called geodetic coordinates - latitude $\left(\phi_{P}\right)$, longitude $\left(\lambda_{P}\right)$, and ellipsoidal height $\left(h_{P}\right)$. The transformation equation between geodetic coordinates and Cartesian coordinates of point $\mathrm{P}\left(\mathrm{X}_{\mathrm{P}}, \mathrm{Y}_{\mathrm{P}}, \mathrm{Z}_{\mathrm{P}}\right)$ can be expressed as (Seeber 2003), 
$\left[\begin{array}{c}X_{P} \\ Y_{P} \\ Z_{P}\end{array}\right]=\left[\begin{array}{c}\left(N_{P}+h_{P}\right) \cos \phi_{P} \cos \lambda_{P} \\ \left(N_{P}+h_{P}\right) \cos \phi_{P} \sin \lambda_{P} \\ {\left[N_{P}\left(1-e^{2}\right)+h_{P}\right] \sin \lambda_{P}}\end{array}\right]$

with

$N_{P}=\frac{a}{\left(1-e^{2} \sin ^{2} \phi_{P}\right)^{\frac{1}{2}}}$

$e^{2}=2 f-f^{2}=\frac{a^{2}-b^{2}}{a^{2}}$

where, $N_{P}$ is the prime vertical radius of curvature at $\mathrm{P}$, and $e$ the first eccentricity of the reference ellipsoid. It is important to state that the different reference systems have been in use and as such, it is necessary to relate them since cartographic products are usually depicted in terms of these systems. One way of relating them is by the method of coordinate transformation between their Cartesian systems which must require the application of Eqn. 1 above. It is required that seven parameters are needed if a complete transformation between two different Cartesian systems based on two different datums is to be carried out effectively and efficiently. The seven parameters comprise the three translational parameters $(\Delta \mathrm{X}, \Delta \mathrm{Y}, \Delta \mathrm{Z})$, which accounts for an origin shift from the geocenter in three-dimensional space, three rotational parameters $(\delta \mathrm{x}, \delta \mathrm{y}, \delta \mathrm{z})$ which accounts for the rotation of the vector position, and the scale factor (ds). For example, if a coordinate vector $X_{1}=\left(x_{1} y_{1} z_{1}\right)^{T}$ (Fig. 3) is represented in one reference frame, the same coordinate can be represented in the second reference frame as $\mathrm{X}_{2}=\left(\mathrm{x}_{2} \mathrm{y}_{2} \mathrm{z}_{2}\right)^{\mathrm{T}}$ by using the following equation (linearized assuming relatively small transformation values)

$X_{2}=X_{1}+T+D X_{1}+R X_{1}$

With

$$
T=\left(\begin{array}{l}
T_{1} \\
T_{2} \\
T_{3}
\end{array}\right) \text { and } R=\left(\begin{array}{ccc}
0 & R_{3} & -R_{2} \\
-R_{3} & 0 & R_{1} \\
R_{2} & -R_{1} & 0
\end{array}\right)
$$

The differential rotation matrix $\mathrm{R}$ is uniform with the positive counterclockwise rotation of axes. In practice, the seven transformation parameters change with time. Thus, differentiating Eqn. 4 above with respect to time can be written:

$$
\Delta X_{2}=\Delta X_{1}+\Delta T+\Delta D X_{1}+D \Delta X_{1}+\Delta R X_{1}+R \Delta X_{1}
$$

$\mathrm{D}$ and $\mathrm{R}$ are of $10^{-5}$ level and $\Delta \mathrm{X}$ is about $10 \mathrm{~cm}$ per year, the terms are $\mathrm{R} \Delta \mathrm{X}_{1}$ are representing $0.1 \mathrm{~mm}$ over 100 years. Therefore, Eqn. 6 can be reduced to (Blick 2004),

$$
\Delta X_{2}=\Delta X_{1}+\Delta T+\Delta D X_{1}+\Delta R X_{1}
$$

\subsection{The WGS 1984 versus Clarke 1880 ellipsoid}

The world Geodetic System 1984 (WGS84) was designed based on the Doppler observations with the original intent of supporting the United States Department of Defense and its Armed forces. It is 
also the nominal datum used by the Global Positioning System (GPS), which was launched by the United States. Several refinements have been applied to this ellipsoid to align it with the International Terrestrial Reference Frame (ITRF), which is regularly updated to help account for the dynamics of the earth. The WGS84 ellipsoid is almost similar to the GRS80 ellipsoid: a geocentric ellipsoid designed to approximate the shape of the geoid on a global scale (Table 1).

The Nigerian geodetic reference ellipsoid is the Clarke 1880 with the center and origin not in coincidence with the Earth's center of mass. The station is roughly located at the center of the primary triangulation network referred to here as the Minna datum. The Minna datum is a

Table 1: Ellipsoid parameters of the Clarke 1880, GRS80, and WGS84 ellipsoids

\begin{tabular}{llllll}
\hline Ellipsoid & $\begin{array}{l}\text { Semi-major axis (a) } \\
(\mathrm{m})\end{array}$ & $\begin{array}{l}\text { Inverse flattening (f- } \\
1)\end{array}$ & Flattening (f) & $\begin{array}{l}\text { Eccentricity } \\
(\mathrm{e} 2)\end{array}$ & $N_{P}($ Eqn. 2) \\
\hline $\begin{array}{l}\text { Clarke } \\
1880\end{array}$ & 6378249.145 & 293.465 & 0.003407561 & 0.006803511 & Varies by $\varphi$ \\
GRS80 & 6378137.000 & 298.257222101 & 0.003352811 & 0.006694380 & Varies by $\varphi$ \\
WGS84 & 6378137.000 & 298.257223563 & 0.003352811 & 0.006694380 & Varies by $\varphi$ \\
\hline
\end{tabular}

local datum with the origin of the coordinate adopted. The control was established using the traditional survey methods, thus resulting in several inherent deficiencies that cause considerable distortion in the network. These include, among others, inaccuracy of the scale factor by compression of the Clarke 1880 ellipsoid. Arguably, these problems lead to, (i) defect in distances measured, (ii) poor definition of the origin of the Nigerian network (iii) absence of geoidal height model, and, (iv) difficulties in the determination of the transformation parameters. However, the Federal Government of Nigeria initiated and pioneered a surveying infrastructure known as the Continuously Operating Reference Station (CORS) across the country, thereby joining the rest of the world to replace the traditional geodetic passive networks, which are the basic infrastructure for Surveying and Mapping in any country. The purpose is to integrate national development with security and defense, which is in line with the government's endeavor to improve its delivery mechanism. The coordination of the CORS stations to the ITRF has become obvious towards the realization of the Geocentric Datum of Nigeria.

\section{Methods}

\subsection{Data used for deriving the transformation parameters}

Data used for the modeling of the transformation parameters between the WGS84 and the Clarke 1880 (Minna) ellipsoid are first-order control points established for orthomapping across Cross River State (a major part of Southern Nigeria). The static differential mode was used in the creation of these control points and is considered suitable forthis research. A total of 107 points whose coordinates in both systems (WGS84 and Minna Datum) were known were used in general. Whereas only 100 points were used in the training and modeling of these parameters, the remaining seven points were used as testing samples and as an index to measure the model performance assessment. A conversion of orthometric heights to ellipsoidal heights was applied for all the points acquired based on the local ellipsoid (Clarke 1880) spheroid to carry out the model of the transformation parameters. 


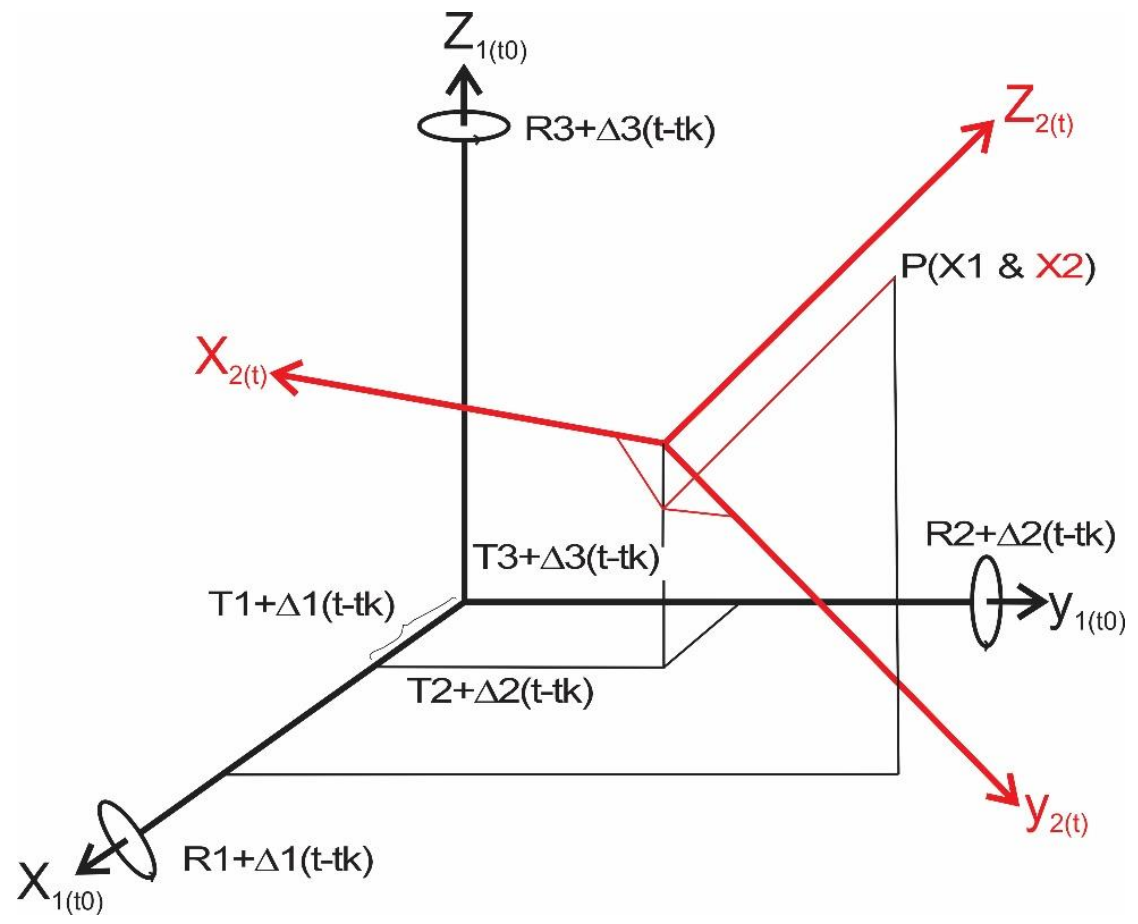

Figure 3: Axes of reference frame.

It was pertinent to distribute these controls around the region to achieve a good network spread for this exercise. This distribution covered the southern part of the region, which includes Bana island, Calabar South, Calabar, and Ikot Nakanda, where approximately, twenty-four (24) controls were established, maintaining an in-range distance of $1.5 \mathrm{~km}$ to $4 \mathrm{~km}$. The northern part of the region which includes, Yala, Abuochiche, Obudu, Ogoja, Obanliku, Ikom, Boki, etc. has an approximate number of fifty (50) controls spread, while maintaining an in-range distance of $4 \mathrm{~km}$ to $6 \mathrm{~km}$. It is significantly larger than the Southern region in terms of landmass. Finally, the central part of the region which includes the Obubra, Effraya, Ugep, Abi, Itigidi, Akpet Central, Biase, Akampa, etc. has an approximate number of forty (40) controls established with an in-range distance of $3 \mathrm{~km}$ to $10 \mathrm{~km}$. In general, these sets of controls provide a means of data relation between datums and if successful, offer a means of control densification in the global system around other parts of Nigeria using them as base stations.

\subsection{The Molodensky-Badekas transformation model}

The Molodensky-Badekas model is a seven-parameter conformal transformation of 3D Cartesian coordinates between two datums. This transformation model is most convenient for the transformation between terrestrial and satellite datums (Krakiswsky et al.1974). The model in its matrix form can be written as;

$\left[\begin{array}{c}X_{W G S 84} \\ Y_{W G S 84} \\ Z_{W G S 84}\end{array}\right]=\left[\begin{array}{l}d X \\ d Y \\ d Z\end{array}\right]+\left[\begin{array}{c}X_{m} \\ Y_{m} \\ Z_{m}\end{array}\right]+\left[\begin{array}{ccc}1+\Delta L & R_{Z} & -R_{Y} \\ -R_{Z} & 1+\Delta L & R_{X} \\ R_{Y} & -R_{X} & 1+\Delta L\end{array}\right]\left[\begin{array}{c}X_{C L K}-X_{m} \\ Y_{C L K}-Y_{m} \\ Z_{C L K}-Z_{m}\end{array}\right]$

With 
$X_{m}=\frac{1}{n} \sum_{i=1}^{n} X_{i} ; Y_{m}=\frac{1}{n} \sum_{i=1}^{n} Y_{i} ; Z_{m}=\frac{1}{n} \sum_{i=1}^{n} Z_{i}$

Where;

$\mathrm{X}_{\mathrm{i}}, \mathrm{Y}_{\mathrm{i}}, \mathrm{Z}_{\mathrm{i}}$ : are the Cartesian coordinates in the local Clarke 1880 system, $n$ is the number of common points. The MB transformation includes three barycentric datum shift parameters ( $\mathrm{dX}, \mathrm{dY}, \mathrm{dZ})$, three rotation elements $\left(R_{X}, R_{Y}, R_{Z}\right)$, and one scale factor $(1+\Delta L)$.

Theoretically, this transformation model can be said to be identical to the Bursa Wolf model. However, the major difference between the two models is the choice of the points about which the axial rotations and scale change are applied (Featherstone 1997).

\subsection{The Bursa Wolf transformation model}

This is another seven-parameter model frequently used for the transformation of 3D Cartesian coordinate between two datums (Kutoglu et al. 2002). However, the Bursa-Wolf transformation model is more suited for the transformation between satellite datums on a global scale. The model in its matrix form is expressed thus;

$\left[\begin{array}{c}X_{W G S 84} \\ Y_{W G S 84} \\ Z_{W G S 84}\end{array}\right]=\left[\begin{array}{c}\Delta X \\ \Delta Y \\ \Delta Z\end{array}\right]+\left[\begin{array}{ccc}1+\Delta L & R_{Z} & -R_{Y} \\ -R_{Z} & 1+\Delta L & R_{X} \\ R_{Y} & -R_{X} & 1+\Delta L\end{array}\right]\left[\begin{array}{l}X_{C L K} \\ Y_{C L K} \\ Z_{C L K}\end{array}\right]$

Where $\mathrm{X}_{\mathrm{WGS} 84}, \mathrm{Y}_{\mathrm{WGS} 84}, \mathrm{Z}_{\mathrm{WGS} 84}$ are the global datum (WGS84) Cartesian coordinates;

$\mathrm{X}_{\mathrm{CLK}}, \mathrm{Y}_{\mathrm{CLK}}, \mathrm{Z}_{\mathrm{CLK}}$ are the local datum (Clarke 1880) Cartesian coordinates.

The BW model similar to the MB model involves three geocentric datum shift parameters $(\Delta \mathrm{X}, \Delta \mathrm{Y}, \Delta \mathrm{Z})$, three rotation elements $\left(\mathrm{R}_{\mathrm{X}}, \mathrm{R}_{\mathrm{Y}}, \mathrm{R}_{\mathrm{Z}}\right)$, and one scale factor $(1+\delta \mathrm{L})$.

\subsection{Computation of ellipsoidal heights for Minna datum}

In order to derive the ellipsoidal height $(h)$ for the sets of the local ellipsoid parameters (Minna datum) provided by "source", the undulations of all 107 points were computed using the 5-parameter Molodensky standard formula (Marzooqi et al. 2005; Nima 2004; Fubara 1995; DMA 1987)

$\Delta h=T_{x} \cos \varphi \cos \lambda+T_{y} \cos \varphi \sin \lambda+T_{z} \sin \varphi-\Delta a\left(\frac{a}{R_{N}}\right)+\Delta f\left(\frac{b}{a}\right) R_{N} \sin ^{2} \varphi$

$\Phi, \lambda=$ WGS84 coordinates of the station; $\mathrm{T}_{\mathrm{x}}, \mathrm{T}_{\mathrm{y}}, \mathrm{T}_{\mathrm{z}}=$ Datum shifts to transform WGS84 datum to Minna datum; $\Delta \mathrm{a}, \Delta \mathrm{f}=$ (Minna minus WGS84) semi-major and flattening respectively. awgs84 = semi-major axis radius of WGS84 ellipsoid $=6378137 \mathrm{~m} ; \mathrm{f}_{\mathrm{WGS} 84}=$ flattening of WGS84 ellipsoid $=1 / 298.257223563$; $\mathrm{a}_{\text {MINNA }}=6378249.145 \mathrm{~m} ; \mathrm{f}_{\mathrm{MINNA}}=1 / 293.465 ; \Delta \mathrm{a}=112.145 \mathrm{~m} ; \Delta \mathrm{f}^{*} 10^{4}=0.54750714(\Delta \mathrm{f}=$ $0.000054750714) ; R_{N}=$ radius of curvature of the prime vertical; $b / a=1-f ; e^{2}=2 f-f^{2}$. Since there were no official transformation parameters as at the time, the values adopted for $T_{x}, T_{y}$, and $T_{z}$ were derived from the procedure in page 207, equations 55 and 56 of Heiskanen et al. (1967) and applied to the origin point of Minna datum:

$$
\begin{aligned}
& T_{x}=a \delta \varphi_{0} \sin \varphi_{0} \cos \lambda_{0}+a \delta \lambda_{0} \cos \lambda_{0} \sin \varphi_{0}-\cos \varphi_{0} \cos \lambda_{0}\left(\delta h_{0}+\delta a+a \delta f \sin ^{2} \varphi_{0}\right) \\
& T_{y}=a \delta \varphi_{0} \sin \varphi_{0} \sin \lambda_{0}+a \delta \lambda_{0} \cos \varphi_{0} \cos \lambda_{0}-\cos \varphi_{0} \sin \lambda_{0}\left(\delta h_{0}+\delta a+a \delta f \sin ^{2} \varphi_{0}\right)
\end{aligned}
$$


$T_{z}=-a \delta \varphi_{0} \cos \varphi_{0}-\sin \varphi_{0}\left(\delta h_{0}+\delta a+a \delta f \sin ^{2} \varphi_{0}\right)+2 a \delta f \sin \varphi_{0}$

Where, $\varphi_{0}, \lambda_{0}$ are latitude and longitude respectively of the origin point; $\delta \varphi_{0}, \delta \lambda_{0}, \delta h_{0}$ are differences between Minna datum and WGS84 datum latitudes, longitudes, and ellipsoidal heights, respectively, of the origin point. $\mathrm{a}=$ semi-major radius of the reference ellipsoid used

$\delta \mathrm{a}, \delta \mathrm{f}=$ difference between the semi-major radius and flattening respectively of the Clarke 1880 ellipsoid (Minna datum) and the WGS84 ellipsoid.

From the computations carried out, the following values were derived for $\mathrm{Tx}, \mathrm{Ty}$ and $\mathrm{Tz} ; \mathrm{Tx}=93.708 \mathrm{~m}$; $\mathrm{Ty}=92.626 \mathrm{~m} ; \mathrm{Tz}=-121.330 \mathrm{~m}$.

The Minna datum ellipsoidal heights were gotten direct from the WGS84 ellipsoidal heights.

\subsection{Least square estimation of the transformation parameters}

The least square adjustment was used for this project where the Bursa Wolf transformation model given above (eqn. 10) can be re-written in a linear form as;

$\left[\begin{array}{l}V_{X} \\ V_{Y} \\ V_{Z}\end{array}\right]=\left[\begin{array}{ccccccc}1 & 0 & 0 & X_{C L K} & 0 & -Z_{C L K} & Y_{C L K} \\ 0 & 1 & 0 & Y_{C L K} & Z_{C L K} & 0 & -X_{C L K} \\ 0 & 0 & 1 & Z_{C L K} & -Y_{C L K} & X_{C L K} & 0\end{array}\right]\left[\begin{array}{c}d X \\ d Y \\ d Z \\ K \\ R_{X} \\ R_{Y} \\ R_{Z}\end{array}\right]-\left[\begin{array}{ccc}X_{W G S 84} & - & X_{C L K} \\ Y_{W G S 84}- & Y_{C L K} \\ Z_{W G S 84} & - & Z_{C L K}\end{array}\right]$

The same procedure can be used to express the Molodensky-Badekas transformation model in its linear form as;

$\left[\begin{array}{l}V_{X} \\ V_{Y} \\ V_{Z}\end{array}\right]=\left[\begin{array}{ccccccc}1 & 0 & 0 & D_{X} & 0 & -D_{Z} & D_{Y} \\ 0 & 1 & 0 & D_{Y} & D_{Z} & 0 & -D_{X} \\ 0 & 0 & 1 & D_{Z} & -D_{Y} & D_{X} & 0\end{array}\right]\left[\begin{array}{c}d X \\ d Y \\ d Z \\ K \\ R_{X} \\ R_{Y} \\ R_{Z}\end{array}\right]-\left[\begin{array}{ccc}X_{W G S 84} & - & X_{C L K} \\ Y_{W G S 84} & - & Y_{C L K} \\ Z_{W G S 84} & - & Z_{C L K}\end{array}\right]$

Where

$\left[\begin{array}{l}D_{X} \\ D_{Y} \\ D_{Z}\end{array}\right]=\left[\begin{array}{lll}X_{C L K} & -X_{M} \\ Y_{C L K} & - & Y_{M} \\ Z_{C L K} & - & Z_{M}\end{array}\right]$

The least square solution for the unknown parameters $(x)$ is computed from;

$\dot{\mathrm{x}}=\left(A^{T} P A\right)^{-1} A^{T} P L$ 
$\sum_{\overline{\mathrm{x}}} \sigma_{0}^{2}\left(A^{T} P A\right)^{-1}$

$$
\sigma_{0}^{2}=\frac{V^{T} P V}{n-u}
$$

Where $V$ is the vector of the residuals, $A$, the design or coefficient matrix, $(\dot{\mathrm{x}}),:$ the vector of unknowns; $L$, the vector of observations; $P$, the weight matrix of the observations; $\sigma_{0}^{2}$, the a-posterior variance of unit weight; $n$, the number of observations; $u$, the number of unknowns.

Where, $A^{T} P A$ is a non-singular matrix called Normal equation coefficient matrix, and $A^{T} P L$ is the normal equation constant (or absolute) term vector. Also, the least squares observation equation for a linear mathematical model is given as:

$$
\begin{aligned}
& A x-b=V \\
& V=A x-b
\end{aligned}
$$

Where $(x)=$ column matrix of the unknown parameters [in this case; $\mathrm{dX}, \mathrm{dY}, \mathrm{dZ} ; \mathrm{Rx}, \mathrm{Ry}, \mathrm{Rz}, \mathrm{K}], b$ is the column matrix of absolute or differences in Cartesian coordinates local Clarke1880 system, $V$, the column matrix of the residuals, A, the design coefficient matrix of the unknown parameters in the observation equation. Attempting to extend the matrix to accommodate a total of 100 control points used for the training, the number of observations has to be determined. Where each point provides 3 observation equations, therefore the total number of observation equations (n) is determined by $3 \times 100$ points. From Eqn. 18, the least squares solution for the unknown parameters (x) including their statistics can be computed. However, the weight (P) in this paper is assumedthe same i.e., unit. Therefore, Eqn. 18 becomes

$\dot{\mathrm{x}}=\left(A^{T} A\right)^{-1} A^{T} L$

The coordinates of the total one hundred and seven points (107) in both systems were used for a preliminary computation of the mean shift parameters, rotation parameters, and the scale factor. This approach prompted an analysis of divergence should the seven testing points be included in the modeling process. However, little or discrepancies were discovered, therefore, the modeling was done using one hundred (100) control points out of the one hundred and seven (107) control points.

\subsection{Model performance assessment}

\subsubsection{Variance factor and standard deviation}

The variance of unit weight for weighted observations (a-posteriori) is given as:

$$
\sigma_{0}^{2}=\frac{V^{T} W V}{n-m}
$$

where $\sigma_{0}^{2}$ is the estimated variance factor, $n$ is the number of observations, $m$ is the number of unknown transformation parameters, hence $n-m$ is the degree of freedom (df), $\mathrm{V}^{\mathrm{T}} \mathrm{WV}$ is

Table 2: Variance and standard deviation for the Bursa Wolf model 


\begin{tabular}{lcc}
\hline Parameters & Variance & Standard Deviation \\
\hline$\Delta \mathrm{X}(\mathrm{m})$ & 0.597415626385789 & \pm 0.77292666 \\
$\Delta \mathrm{Y}(\mathrm{m})$ & 4.017099042837800 & \pm 2.00427020 \\
$\Delta \mathrm{Z}(\mathrm{m})$ & 1.112261713384037 & \pm 1.05463819 \\
$\Delta \mathrm{x}\left({ }^{\circ}\right)$ & 0.000000000000016 & $\pm 1.249 \times 10^{-7}$ \\
$\Delta \mathrm{y}\left({ }^{\circ}\right)$ & 0.000000000000026 & $\pm 1.6125 \times 10^{-7}$ \\
$\Delta \mathrm{z}\left({ }^{\circ}\right)$ & 0.000000000000099 & $\pm 3.1464 \times 10^{-7}$ \\
$\Delta \mathrm{s}$ & 0.000000000000014 & $\pm 1.1832 \times 10^{-7}$ \\
\hline
\end{tabular}

Table 3: Variance and standard deviation for the Molodensky Badekas model

\begin{tabular}{lcc}
\hline Parameters & Variance & Standard Deviation \\
\hline$\Delta \mathrm{X}(\mathrm{m})$ & 5.919972057953653 & \pm 2.43310501 \\
$\Delta \mathrm{Y}(\mathrm{m})$ & 5.980999287880334 & \pm 2.44560830 \\
$\Delta \mathrm{Z}(\mathrm{m})$ & 5.919972057953653 & \pm 2.43308446 \\
$\Delta \mathrm{x}\left({ }^{\circ}\right)$ & 0.000000000019226 & \pm 0.000004385 \\
$\Delta \mathrm{y}\left({ }^{\circ}\right)$ & 0.000000001558587 & \pm 0.00003947 \\
$\Delta \mathrm{z}\left({ }^{\circ}\right)$ & 0.000000005539887 & \pm 0.00007443 \\
$\Delta \mathrm{s}$ & 0.000000000866926 & \pm 0.00002944 \\
\hline
\end{tabular}

the weighted sum of the residuals. A good procedure for analyzing error involves finding the variance factor which can be further used to compute for the standard deviation of the observation. Where the standard deviation of the observation can be gotten by finding the square root of the variance but without the weight element.

$$
\sqrt{\sigma_{0}^{2}}=\sqrt{\frac{V^{T} V}{n-m}}
$$

\subsubsection{Accuracy determination by variance-covariance estimation}

The variance-covariance matrix is the product of the unit variance and the inverse of the normal matrix, given the equation,

$$
C_{X}=\sigma_{0}^{2}\left(A^{T} A\right)^{-1}=\sigma_{0}^{2} Q
$$

The root mean square errors from the various considerations formed the basis for deciding on the optimum results.

\subsubsection{Root Mean Square error}

In assessing the performance of the developed model,the error function (RMS) for both models was computed using the set of transformation parameters obtained from the estimation to transform the WGS84 to Minna datum Cartesian coordinates. This root mean square error from the various considerations formed the basis for deciding on the optimality of theresults. 


$$
r m s^{2}=\sum_{i=1}^{N}\left[\left(x_{i}-X T_{i}\right)^{2}+\left(y_{i}-Y T_{i}\right)^{2}+\left(z_{i}-z T_{i}\right)^{2}\right]
$$

where $x$ y $z$ represents Cartesian coordinates of points on either WGS84 or Minna Datums while $X T$ YT ZT denotes transformed WGS84 Cartesian coordinates of the same point on the Minna Datum or vice versa.

(a)

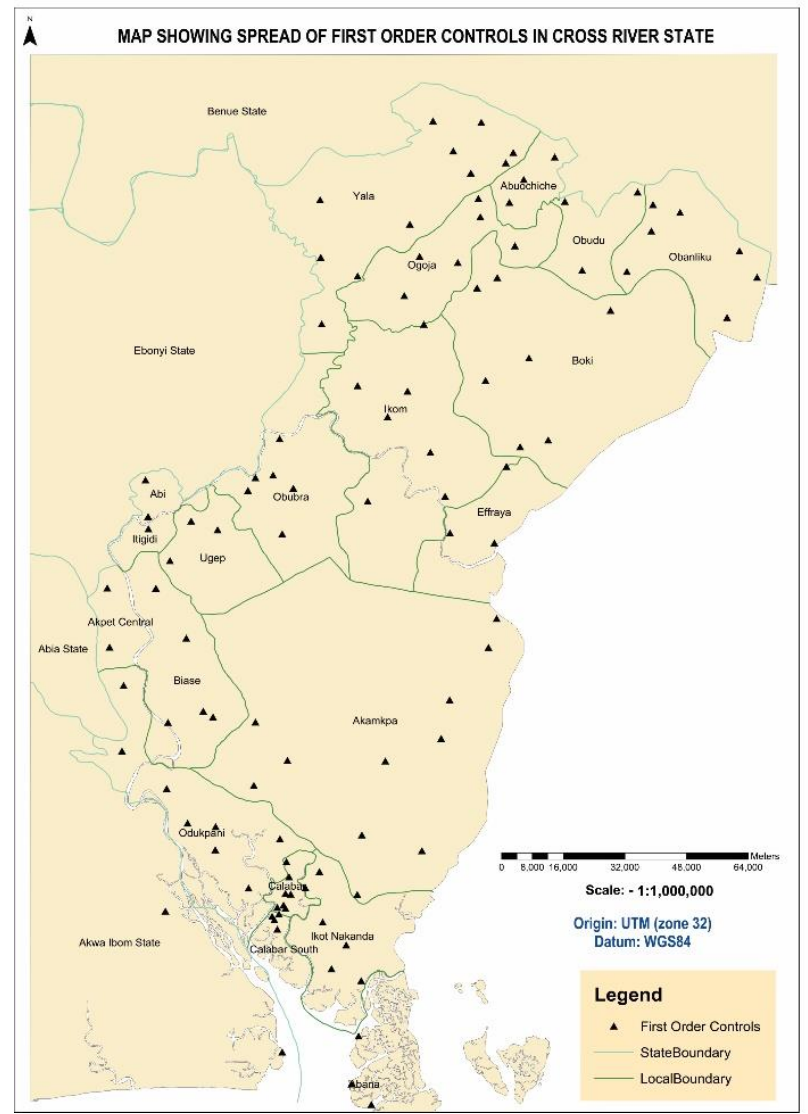

(b)

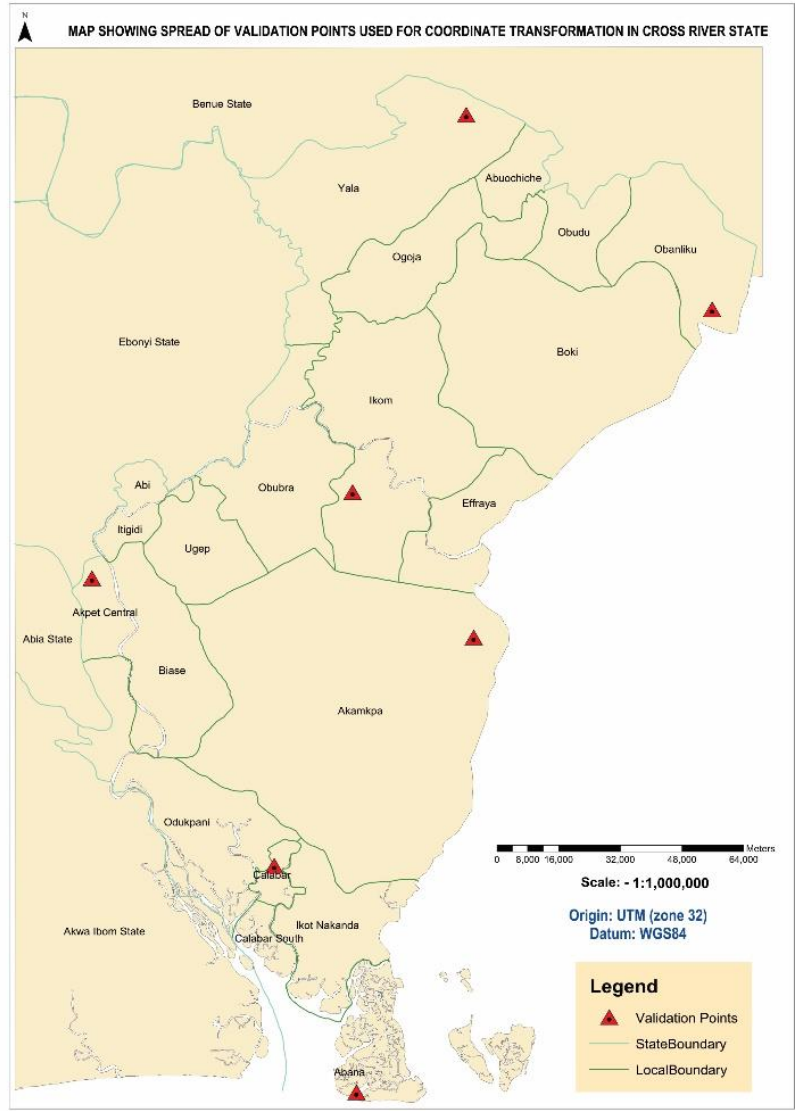

Figure 4: Spatial distribution of 107 points used for developing the models and the spread of the 7 points used for the validation exercise.

The computation of the absolute and relative error and accuracy of the transformation is mostly dependent on the values derived in the variance-covariance estimation. It was therefore applied in this estimation to derive the results in Tables 4 and 5, which are the covariance matrices of both models. 


\section{Results and discussion}

In Nigeria, it is periodically required to transform point coordinates between the conventional earthcentered frame based on the WGS84 ellipsoid, and the old local geodetic coordinate frame based on the Minna datum which uses Clarke 1880 as a reference ellipsoid. However, the possibility to transform coordinates from one datum to another comes with the knowledge of the relationship between them (Eqn. $8,10)$.

Table 4: Covariance matrix of the Bursa Wolf model

\begin{tabular}{|lllllll|}
\hline 0.5974 & -0.3893 & 0.1353 & 0.0000 & -0.0000 & -0.0000 & -0.0000 \\
\hline-0.3893 & 4.0171 & -1.2816 & -0.0000 & 0.0000 & 0.0000 & -0.0000 \\
\hline 0.1353 & -1.2816 & 1.1123 & 0.0000 & -0.0000 & -0.0000 & -0.0000 \\
\hline 0.0000 & -0.0000 & 0.0000 & $1.6 \times 10-14$ & -0.0000 & -0.0000 & 0.0000 \\
\hline-0.0000 & 0.0000 & -0.0000 & -0.0000 & $2.6 \times 10-14$ & 0.0000 & -0.0000 \\
\hline-0.0000 & 0.0000 & -0.0000 & -0.0000 & 0.0000 & $9.9 \times 10-14$ & -0.0000 \\
\hline 0.0000 & -0.0000 & -0.0000 & -0.0000 & 0.0000 & 0.0000 & $1.4 \times 10-14$ \\
\hline
\end{tabular}

Table 5: Covariance matrix of the Molodensky Badekas model

\begin{tabular}{ccccccc}
\hline-5.9200 & -0.0000 & 0.0000 & -0.0000 & -0.0000 & -0.0000 & -0.0000 \\
\hline 0.0038 & -5.9810 & 0.0000 & -0.0000 & -0.0000 & -0.0000 & -0.0000 \\
\hline 0.0000 & 0.0000 & -5.9199 & 0.0000 & 0.0000 & 0.0000 & 0.0000 \\
\hline-0.0000 & -0.0000 & 0.0000 & $-1.9 \times 10-11$ & -0.0000 & -0.0000 & -0.0000 \\
\hline-0.0000 & -0.0000 & 0.0000 & -0.0000 & $-1.6 \times 10-9$ & -0.0000 & -0.0000 \\
\hline-0.0000 & -0.0001 & 0.0000 & -0.0000 & -0.0000 & $-5.54 \times 10-9$ & -0.0000 \\
\hline-0.0000 & -0.0000 & 0.0000 & -0.0000 & -0.0000 & -0.0000 & $-8.67 \times 10-10$ \\
\hline
\end{tabular}

For the purpose of comparison, the Bursa Wolf (Eqn. 10), which is best suited for the transformation of two satellite-derived datum on a global scale, and the MolodenskyBadekas (Eqn. 8), which is best suited for the transformation between local and global datums were used. Their variance factor and standard deviation were determined to ascertain their respective degrees of acceptability for this study.

\subsection{Mode of derivation of the transformation parameters}

The sets of similarity transformation parameters for both models were derived by analyzing a set of survey control points with their coordinates known in both datums (Global and local). The minimum number of controls required for this exercise is solely dependent on the model in question and there is no limit to the number of coordinates to be used. However, for this exercise, a total of one hundred and seven points (Fig. 4) were used. From this amount, one hundred points were used to develop the models and the remaining seven points were used as a basis to test their accuracies. 
Table 6a: Results for the Bursa Wolf Model

\begin{tabular}{|lll|}
\hline PARAMETERS & BW MODEL & STD. DEV \\
\hline $\mathrm{X}(\mathrm{m})$ & -528.8544272599975 & \pm 0.77292666 \\
$\Delta \mathrm{Y}(\mathrm{m})$ & -164.5823217735015 & \pm 2.00427020 \\
$\Delta \mathrm{Z}(\mathrm{m})$ & 79.3935380631010 & \pm 1.05463819 \\
$\Delta \mathrm{x}(\mathrm{o})$ & -0.0000239464420 & $\pm 1.249 \times 10-7$ \\
$\Delta \mathrm{y}(\mathrm{o})$ & -0.0000137330777 & $\pm 1.6125 \times 10-7$ \\
$\Delta \mathrm{Z}(\mathrm{o})$ & -0.0000158722030 & $\pm 3.1464 \times 10-7$ \\
$\Delta \mathrm{s}$ & 0.0000694294654 & $\pm 1.1832 \times 10-7$ \\
Variance & $4.1108873244898 \mathrm{E}-7$ & \\
SD & 0.0006411620173162 & \\
SEM & 0.000171357757015 & \\
C.I $(90 \%)$ & $1.645 \overline{\mathrm{x}}$ & \\
Sum & -0.0010606 & \\
\hline
\end{tabular}

Table 6b: Results for the Molodensky Badekas Model

\begin{tabular}{|lll|}
\hline PARAMETERS & MB MODEL & STD. DEV \\
$\Delta \mathrm{X}(\mathrm{m})$ & 99.344640001658689 & \pm 2.43310501 \\
$\Delta \mathrm{Y}(\mathrm{m})$ & 14.883071267146580 & \pm 2.44560830 \\
$\Delta \mathrm{Z}(\mathrm{m})$ & -60.279356996844790 & \pm 2.43308446 \\
$\Delta \mathrm{x}(\mathrm{o})$ & -0.0000239464420 & \pm 0.000004385 \\
$\Delta \mathrm{y}(\mathrm{o})$ & 0.000021346404411 & \pm 0.00003947 \\
$\Delta \mathrm{z}(\mathrm{o})$ & 0.000035924462804 & \pm 0.00007443 \\
$\Delta \mathrm{s}$ & -0.00006984762664 & \pm 0.00002944 \\
Variance & $5.4020152061224 \mathrm{E}-7$ & \\
SD & 0.00073498402745382 & \\
SEM & 0.0001964327439631 & \\
C.I $(90 \%)$ & $1.645 \overline{\mathrm{x}}$ & \\
Sum & 0.000811 & \\
\hline
\end{tabular}

\subsection{Efficiency and accuracy of both models}

A well-established method of assessing the accuracy of a transformation model is by comparing transformed coordinates at designated points with observed coordinates defined in both datums and reading their respective degree of correlation. Consider the typical situation which was adopted in this research; Coordinate set 1 which has been computed from network 1 in Datum 1; Coordinate set 2 which has been computed from network 2 in Datum 2. If the coordinates from set 1 are converted to Datum 2, they will generally not match the coordinate of set 2 exactly. This is because both coordinate sets are derived via different measurement sets or network geometries (e.g., Network 1 may have been measured by classical triangulation and Network 2 by GPS). This will therefore result in one network appearing offset and distorted relative to the other (Blick 2004). The differences between the transformed Datum 1 


\section{Discrepancy level of validation points}
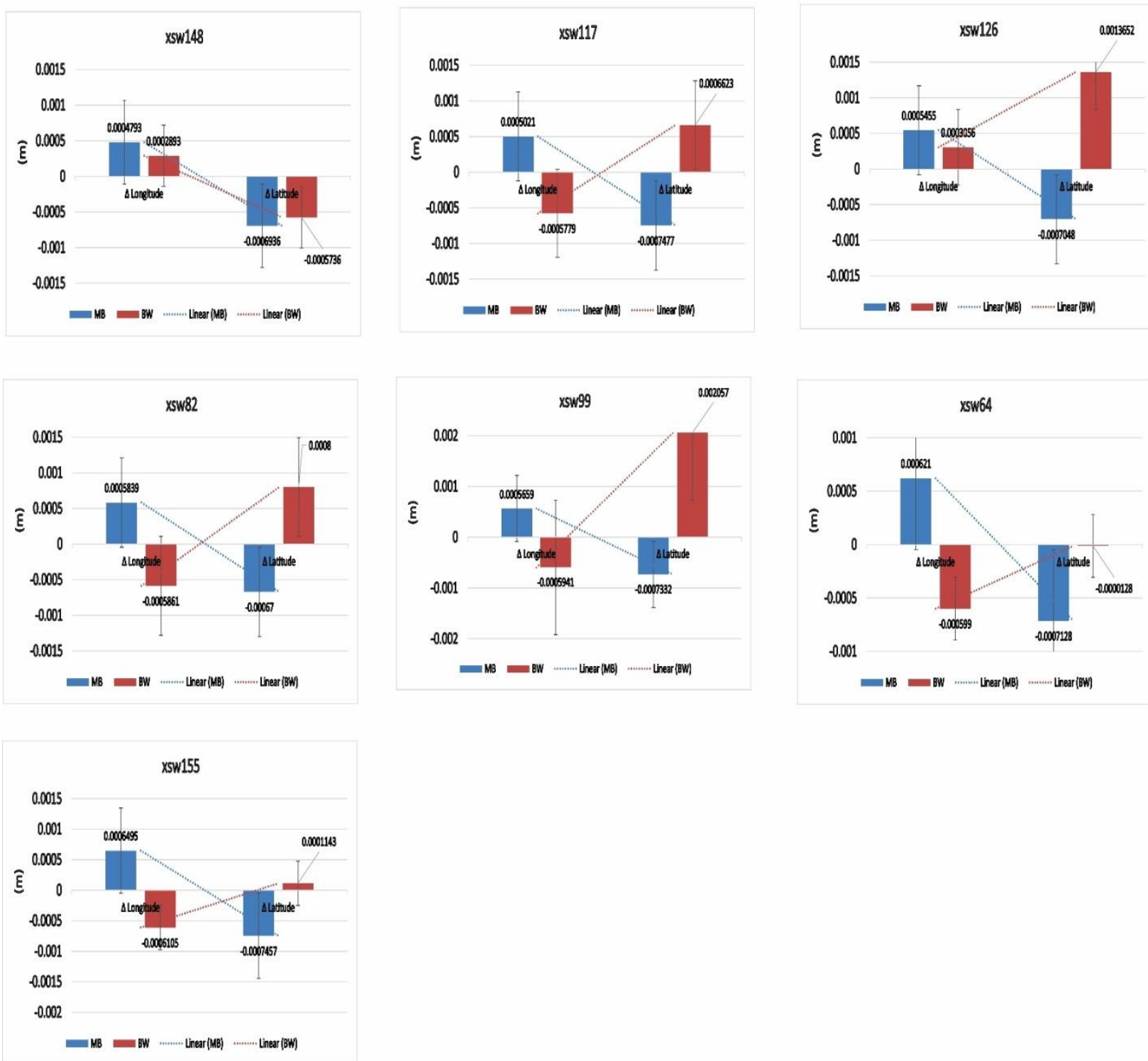

Figure 5: Degree of correlation between the BW (Bursa Wolf) and MB (Molodensky-Badekas) models when tested on the seven validation points for selected locations.

coordinates and the network Datum 2 coordinates are known as residuals. Their magnitude indicates the quality of the network held in the two datums, as well as an indication of the accuracy of the transformation between those datums (Blick 2004). To check the efficiency of the Bursa Wolf and MolodenskyBadekas models produced, the residuals between both models were estimated. It was pertinent to validate the models by applying them on the training set, which included seven control points spread out across the region. These controls include, xsw148, xsw117, xsw126, xsw97, xsw82, xsw64, xsw155 (Fig. 4). Hence, the objective here is to ascertain the extent of accuracy achievable for a particular model. It can be seen that the accuracy level is acceptable (Table 7) as the values of the result show a minimal deviation from the average of both systems (models). The differences between the observed and 
computed coordinates were developed for both systems as a form of a standard check for conformity (Table 7).

Table 7: Difference between observed and computed WGS84 coordinates of the validation points. MB is Molodensky Badekas model while BW is the Bursa Wolf model.

\begin{tabular}{|c|c|c|c|}
\hline Validation Points & Model & Lat. Diff (Degrees) & Long. Diff (Degrees) \\
\hline \multirow[t]{2}{*}{ xsw148 } & B W & 0.0004793 & -0.0006936 \\
\hline & M B & 0.0002893 & -0.0005736 \\
\hline \multirow[t]{2}{*}{ xsw 117} & B W & 0.0005021 & -0.0007477 \\
\hline & M B & -0.0005779 & 0.0006623 \\
\hline \multirow[t]{2}{*}{ xsw 126} & B W & 0.0005455 & -0.0007048 \\
\hline & M B & 0.0003056 & 0.0001365 \\
\hline \multirow[t]{2}{*}{ xsw 99} & B W & 0.0005839 & -0.0006700 \\
\hline & M B & -0.0005861 & 0.0008000 \\
\hline \multirow[t]{2}{*}{ xsw 82} & B W & 0.0005659 & -0.0007332 \\
\hline & M B & -0.0005941 & 0.002057 \\
\hline \multirow[t]{2}{*}{ xsw 64} & B W & 0.0006210 & -0.0007128 \\
\hline & M B & -0.0005990 & -0.0000128 \\
\hline \multirow[t]{2}{*}{ xsw 155} & B W & 0.0006495 & -0.0007457 \\
\hline & M B & -0.0006105 & 0.0001143 \\
\hline
\end{tabular}

\subsection{Significance of the newly developed models}

The use of the newly developed regional transformation parameters derived from this research has shown an improvement of $68 \%$ in terms of the correlation between expected and derived coordinates in the validation points against the official transformation parameters that have been in use by the region for some years now. The Molodensky Badekas provided considerably strong agreement between the expected and derived coordinates (Fig. 6), which prompted the use of the transformation model on other 
points in the region that were not part of the original exercise. Two second-order points whose coordinates were known in both the global and local systems were used and a 14\% reduction in accuracy was obtained (Eqn. 27). Notably, the drops in accuracy level were attributed to the triangulation mode used for the data acquisition of the points in the local system and the official transformation parameters used to derive the global coordinates of the two points. Regardless of the difference in the parameter set of the official transformation model and the regional transformation model obtained from this study, the coordinate differences recorded during the phase of validation (Fig. 6) show that the adopted parameters are largely accurate. In addition to this, the differences in the model seven datum transformation parameters is based on the difference in the normal and coefficient matrices. This modeling was undertaken for a major part of Nigeria with moderately high distribution of control points. It is expected that the accuracy achieved using the regional transformation as obtained in this study will be equivalent to the official transformation parameters for the entire country as the accuracy achieved is largely a factor of the size of the area, the number of controls used and the spatial distribution of these control points. However, if relevant corrections are applied, the achieved parameters could be used for other academic exercises and further research.

Coordinate difference between observed and computed validation points
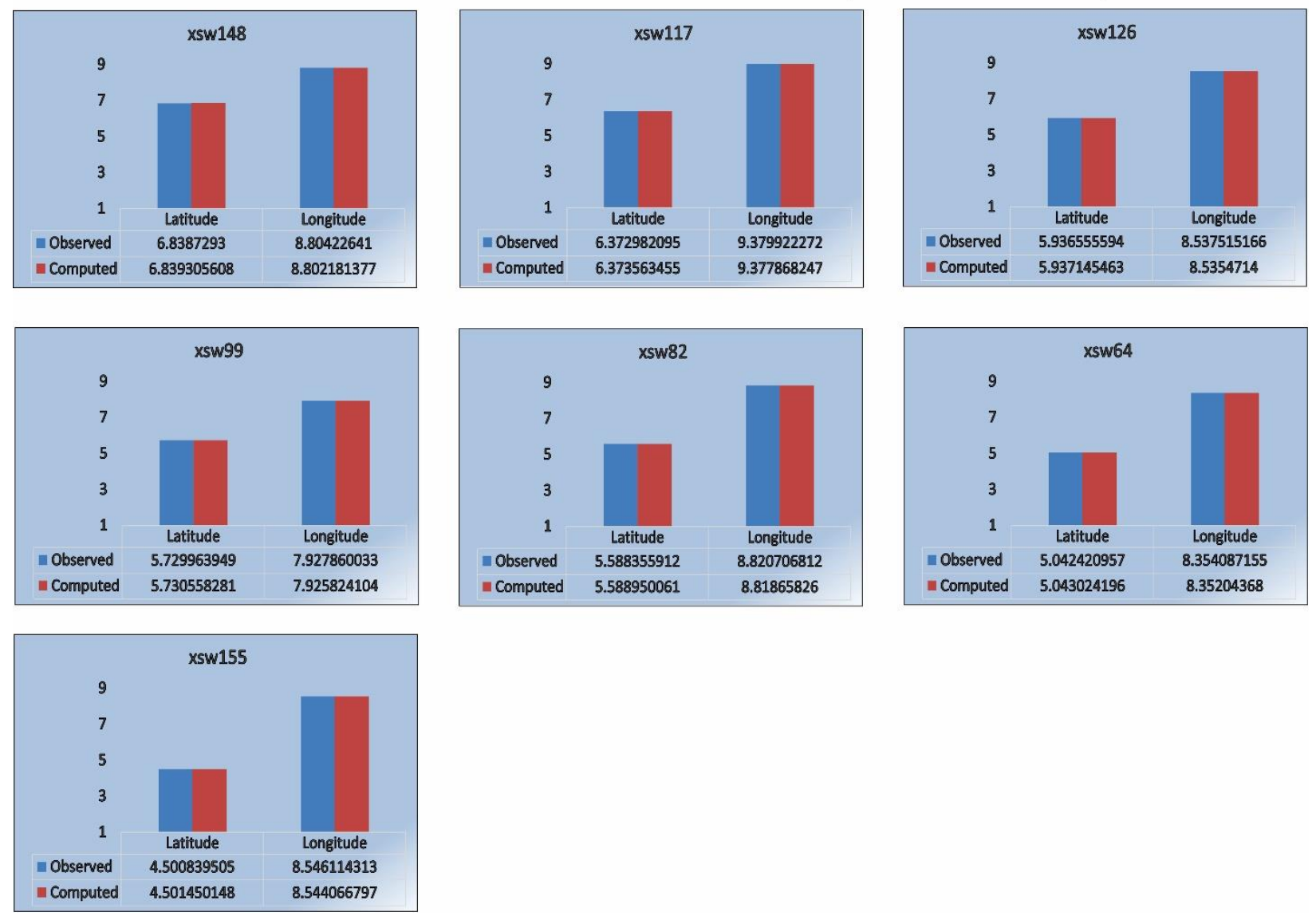

Figure 6: Chart showing longitude and latitude difference between expected and computed validation points. Where, the observed and computed values represent the geographic coordinate of the measured and transformed points respectively. 
In this study, the Bursa Wolf and Molodensky Badekas models were used as an optimum criterion to aid in selecting the model that will be most effective and suitable in the transformation between the global datums (WGS84) and the Nigerian local datum (Minna Datum). These statistical indices are capable of quantifying the error magnitude produced by the estimation in the validation phase. Hence, the closer the statistical indices are to zero (Table 7) the better the prediction capabilities of the method. In the comparison between the correlation relationships of the two models, it is shown (Fig. 5) that the highest discrepancy between the BW and MB models was point xsw155 with a latitude range of $0.126 \mathrm{~cm}$ and point xsw177 with a longitude range of $0.141 \mathrm{~cm}$. The estimated standard deviation, standard error of the mean (SEM), and sum values show a practical expression for the precision of the outputs from the BW (Table 6a) and MB (Table 6b) models. In Table 6a, it was shown that the Bursa Wolf model has a superior dispersion network to the mean compared to the Molodensky Badekas model (Table 6b). This could be largely attributed to the fact that the Bursa Wolf model had the least standard deviation values. The standard deviation further indicates that in the case of the Bursa Wolf model, the limit of the error bound by which every value within the predicted projected grid coordinates varies from its most probable value (mean) is reduced but this does not mean that the Bursa Wolf model provided a better transformation than the Molodensky Badekas model.

A good way of checking a better transformation model is the minimal difference that is derived from summing the validation differences hence, the closer the sum is to zero, the more accurate the model. Considering the variance, standard deviation, standard error of the mean (SEM), and confidence values, it was uncovered that the Bursa Wolf model outperformed the MB model in the terms of network stability (only of the validation points). For the most important parameter, which is the sum; indicatingthe degree of closeness of each derived parameter to its corresponding expected value, the MB models showed a better prediction and substantial association as their values were closer to the measured data than the Bursa Wolf model. The interpretation made in line with the differences between the observed and computed coordinates of the validation points (Table 7) shows that the minimum deviation for the Bursa Wolf model coordinate difference is 0.0004793 whereas Molodensky Badekas model showed a minimum deviation of -0.0000128 . This further indicates the accuracy range of these two methods because these differences ideally should be 0 . However, this could only be realized if both the local/terrestrial network were perfect. Such a situation is considerably difficult to achieve and is therefore a key explanation as to why coordinate transformation may not be the ultimate solution to datum problems (Newsome et al. 2003).

For the computation of these parameters, a total of 100 points, which were used for this exercise were evenly distributed across the study area (Fig. 4). This promised to improve the accuracy achievable around the region of interest. This same factor was put into consideration while selecting the points which would be used for the validation of this exercise. The points chosen for the validation exercise were thus distributed to the north, south, east, west, and central parts of the region to provide a good network for the testing of the derived model/parameters. The validation results showed that both the Molodensky Badekas and Bursa Wolf models gave similar results. However, from the results of the model performance assessment, the Molodensky Badekas method seems suitable for the transformation of coordinate from the Minna datum to the WGS84. This is to be expected as this model is best applied in the transformation from global tothe local system and vice versa whereas the Bursa Wolf model is best suited for transformation between two satellite-derived datum on a global scale. Since the transformation parameters to be used for geodetic applications could be used by non-geodesist for several practical applications, Kutoglu et al. (2002) recommended the use of Molodensky Badekas model instead of the 
Bursa Wolf model to normalize practice especially in transformations containing local networks. As with increasingly interesting applications of mathematical models and machine learning in addressing critical environmental problems such as global warming, floods, and groundwater hydrology (Anupam and Pani 2020; Ndehedehe and Ferreira 2020;Mogaji and Lim 2019), these applications are also gradually emerging in coordinate transformation.

Support Vector Machine and Artificial Neural Network, for example, have shown potentials in coordinate transformation. The emergence of a deep learning architecture to improve coordinate transformation (Gin et al. 2020) are indications of interesting future research directions that can be leveraged upon for effective geodetic transformations between systems.

\section{Conclusions}

The optimal transformation parameters set was computed for a major part of southern Nigeria between WGS84 datum and the Clarke 1880 (Minna datum). The use of the newly developed regional transformation parameters derived from this study has shown an improvement of $68 \%$ in terms of the correlation between expected and derived coordinates in the validation points against the official transformation parameters, that have been in use by the region for some years now. The Molodensky Badekas model provided considerably strong agreement between the expected and derived coordinates which prompted the use of the transformation model on other points in the region which were not part of the original exercise. The full seven parameters set is recommended for transformation using both models. The parameters were estimated using a total of 100 points, which were sufficiently well distributed over the study area including the outskirts of the region. The data conversions using the above parameters were tested against seven points which were not part of the original computation. The availability of the precise geoid model makes it possible to compute a unique set of transformation parameters for the region. For any point being transformed from the local to the global system or vice versa, a precision of $10-100 \mathrm{~cm}$ can be derived considering both models (Table 7). It should be noted that the transformation parameters

to be determined for any geodetic application could be made use by any non-geodesist for various applications and purposes. For this purpose, to unify and normalize practice, Molodensky Badekas over Bursa Wolf is recommended, especially in transformations between local and global networks.

\section{Conflict of interest declaration}

The authors declare that they have no known competing financial interests or personal relationships that could have appeared to influence the work reported in this paper.

\section{Acknowledgments}

The author is grateful to the Cross River state government, Nigeria for the data used in this study. 


\section{References}

Anupam S, Pani P (2020) Flood forecasting using a hybrid extreme learning machine-particle swarm optimization algorithm (ELM-PSO) model. Model. Earth Syst. Environ. 6, 341-347.

https://doi.org/10.1007/s40808-019-00682-z

Ayer J, Tiennah T (2008) Datum Transformation by the Iterative Solution of the Abridging Inverse Molodensky Formulae. The Ghana surveyor, Vol. 1, no. 2, pp. 59-66

Blick G (2004) Reference frames in practice manual. International Federation of Surveyors. https://www.fig.net/resources/publications/figpub/pub64/Figpub64.pdf Accessed 10 Jan 2020

Dawod GM, Mirza NM, Al-Ghamdi AK (2010) TS 8 Simple Precise Coordinates Transformations for Geomatics Applications in Makkah Metropolitan Area, Saudi-Arabia. Bridging the Gap between Cultures FIG Working Week, Marrakech, Morocco:18-22

Deakin R. (2006) A note on the bursa-wolf and molodensky-badekas transformations. http://www.mygeodesy.id.au/documents/Similarity\%20Transforms.pdfAccessed14 January 2020

Devi S, Mishra RP (2020) a mathematical model to see the effects of increasing environmental temperature on plant-pollinator interactions. Model. Earth Syst. Environ. 6, 1315-1329. https://doi.org/10.1007/s40808-020-00763-4

Dzidefo A (2011) Determination of Transformation Parameters between the World Geodetic System 1984 and the Ghana Geodetic Network. Master's Thesis, Department of Civil and Geomatic Engineering, Kwame Nkrumah University of Science and Technology, Kumasi, Ghana

DMA (1987) Department of defence world geodetic system 1984: Its definition andrelationshipwithlocalgeodeticsystems ( $2{ }^{\text {nd }}$ Edition $)$.DefenceMappingAgency, Washington.TechnicalRepo rt no.8350.2.

Featherstone WE (1997) A comparison of existing coordinate transformationmodels andparametersinAustralia.Cartography,26(1):13-25.doi:10.1080/00690805.1997.9714042

Featherstone W E, Galloway D, Goulding P,Reit BG (1999)Transformation between Australian datums using a modified transverse Mercator projection.Cartography, 28(1):19-

31.doi:10.1080/00690805.1999.9714297

FubaraJ M D (1995)Determination of 7-parameters datum transformation forShell PetroleumDevelopmentCompany;foruseintheSouthernNigeria.GeodeticSurveysNigeria.

Gin C, Lusch B, Brunton B,Kutz J (2020) Deep learning models for global coordinate transformations that linearise PDEs. European Journal of Applied Mathematics, 1-25.

doi:10.1017/S0956792520000327

Heiskanen W A, Moritz H (1967) Physical geodesy. W.H.Freeman and Co., SanFrancisco. 
ICSM (2002) Geocentric datum of Australia technical manual, version 2.2. ANZLIC Committee on Surveying and Mapping.

www.icsm.gov.au/icsm/gda/gdatm/index.html on 10 Jan2020 Accessed 10 January 2020

Janssen V (2009) Understanding coordinate systems, datums and transformations in Australia.in:

Ostendorf, B.,Baldock, p., Bruce, d., Burdett, M. and P. Corcoran (eds.).

ProceedingsoftheSurveying\&SpatialSciencesInstituteBiennialInternationalConference,Adelaide 2009,

Surveying \& Spatial Sciences Institute, pages697-715.

https://www.researchgate.net/publication/228649896 Understanding coordinate systems datums and mation s in Australia Accessed 10 January 2020

Kinneen R W, Featherstone WE (2004)

AnempiricalcomparisonofcoordinatetransformationsfromtheAustraliangeodeticdatum(AGD66andAGD84 )to thegeocentricdatumofAustralia(GDA94).Journal of Spatial Science, 49(2):1-29

doi:10.1080/14498596.2004.9635019.

KrakiwskyEJ,ThomsonDB(1974)Mathematicalmodelsforthecombination of terrestrial and satellite networks. The Canadian Surveyor, 28(5):606-615

doi:10.1139/tcs-1974-0105

Kutoglu H S, Mekik C, Akcin H (2002) A comparison of two well known models for 7-parameter transformation.AustralianSurveyor, 47(1):24-30.

doi:10.1080/00050356.2002.10558839

Marzooqi A Y, F H Ahmed, S.I (2005) Derivation of datumtransformation parameters for dubai emirate. from Pharohs to geoinformatics. FIG. FIG Working Week and GSDI-8 Cairo, Egypt April15-21

Mogaji KA, Lim HS(2020) A GIS-based linear regression modeling approach to assess the impact of geologic rock types on groundwater recharge and its hydrological implication. Model. Earth Syst. Environ. 6, 183-199https://doi.org/10.1007/s40808-019-00670-3

NdehedeheCE, Ferreira VG. (2020). Assessing land water storage dynamics over South America, Journal of Hydrology, 580,124339.

Newsome G G, Harvey B R (2003) GPS coordinate transformation parameters for Jamaica. Survey Review, 37(289):218-234 doi:10.1179/sre.2003.37.289.218

NIMA (2004) Department of defense world geodetic system 1984: Its definition and relationshipswithlocalgeodeticsystems, technical report 8350.2.

http://earth-info.nga.mil/GandG/publications/tr8350.2/tr8350_2.html Accessed 10 January 2020

Okeke F (2013) Coordinate transformation parameters and procedure for Nigeria. Final

WorkshopontheAdoptionofTransformationParametersforNigeriaheldon2-3July 2013, Abuja

Seeber G (2003) Satellite geodesy, second ed. Walter de Gruyter, Berlin, pg 589

Tierra A, Dalazoana R, Freitas S D (2008) Using an artificial neuralnetworkto improve thetransformation of coordinates between classical geodetic reference frames. Computers \& Geosciences, 34(3): 181 189doi.org/10.1016/j.cageo.2007.03.011 
Vanicek P, Krakiwsky E (1986) Geodesy: The concepts, 2nd ed. North Holland,Amsterdam

ZiggahYevenyo, Yao \&Youjian, Hu \&Amans, Christian \& Fan, Dong. (2013) Determination of GPS Coordinate Transformation Parameters of Geodetic Data between Reference Datums-A Case.

International Journal of Engineering Sciences and Research Technology. Vol. 2.956

Ziggah Y Y, YoujianH,Tierra A R, Laari P B (2019) Coordinate Transformation between Global and Local Data Based on Artificial Neural Network with K-Fold Cross-Validation in Ghana. Earth Sciences Research Journal, 23(1), 67-77 doi: https://doi.org/10.15446/esrj.v23n1.63860

Ziggah Y Y, Hu Y, Issaka Y \& Laari P B (2019)b. Least squares support vector machine model for coordinate transformation. Geodesy and Cartography, 45(1), 1627.

https://doi.org/10.3846/gac.2019.6053 